\title{
Antitumor activity of YM155, a selective survivin suppressant, in combination with cisplatin in hepatoblastoma
}

\author{
YING YU ${ }^{1}$, XIAOSU ZHAO ${ }^{3}$, YU ZHANG ${ }^{3}$, YANLING KANG $^{3}$, JIAQI WANG ${ }^{3}$ and YINGCHUN LIU $^{2}$ \\ Departments of ${ }^{1}$ Hepatobiliary and Pancreatic Surgery, ${ }^{2}$ Pediatric Surgery, The First Hospital of Jilin University, Changchun, \\ Jilin 130021; ${ }^{3}$ The Nursing Department of the First Hospital of Jilin University, Changchun, Jilin 130021, P.R. China
}

Received December 23, 2014; Accepted March 12, 2015

DOI: 10.3892/or.2015.3947

\begin{abstract}
Cisplatin (CDDP) is a chemotherapeutic drug that is often used for the treatment of hepatoblastoma. However, many patients acquire resistance to therapeutic agents leading to local and distant treatment failure. It has been shown that suppression survivin contributed to the inhibition of tumor growth and enhanced chemotherapeutic sensitivity in several types of cancer. The aim of the present study was to determine whether treatment with sepantronium bromide (YM155), a novel small molecule inhibitor of survivin, enhanced the sensitivity of CDDP to hepatoblastoma cells, leading to the therapeutic efficacy of cisplatin. In vitro and in vivo models were used to examine the anticancer efficacy of YM155, either as a monotherapy or in combination with CDDP to identify more effective therapeutics against hepatoblastoma. The results showed that survivin expression was upregulated in hepatoblastoma tissues and cell lines, and that YM155 inhibited survivin expression in hepatoblastoma cells in a dose-dependent manner. YM155 enhanced sensitivity of CDDP to human HepG2 and HuH-6 hepatoblastoma cells. The YM155 combination with CDDP in hepatoblastoma cells significantly decreased cell proliferation and formation, and induced cell apoptosis than either agent alone. In a mouse xenograft model, YM155 combined with CDDP significantly suppressed tumor growth compared to the monotherapy. Taken together, these findings suggested that the combination of YM155 and CDDP is a promising drug candidate for the treatment of hepatoblastoma.
\end{abstract}

\section{Introduction}

Hepatoblastoma is the most frequently diagnosed malignant liver tumor in children (1), accounting for $~ 50 \%$ of the liver tumors and $1.3 \%$ of malignant tumors in children (2). A recent

Correspondence to: Professor Yingchun Liu, Department of Hepatobiliary and Pancreatic Surgery, The First Hospital of Jilin University, Changchun, Jilin 130021, P.R. China

E-mail: liuyingchun568@sina.cn

Key words: hepatoblastoma, cisplatin, YM155, survivin pediatric cancer epidemiological analysis showed that the average annual percentage in the incidence of hepatoblastoma has been on the increase in the last 30 years (3). Despite the fact that surgical therapy combined with chemotherapy has improved the prognosis of patients with hepatoblastoma, disease-free survival rates remain $<50 \%$ for patients with advanced hepatoblastoma, especially the patients with distant metastases (cancer spreads to other organs and tissues of the body) (4). Cisplatin (CDDP) is a chemotherapeutic agent that is often used for the treatment of hepatoblastoma (5). However, many patients acquire resistance to chemotherapeutic agents, which resulted in treatment failures (6). Therefore, understanding the molecular mechanisms that contribute to drug resistance is crucial to optimize therapeutic options or develop novel effective therapy for hepatoblastoma.

Survivin, an apoptotic inhibitor, is involved in the suppression of apoptosis and regulation of mitosis in cancer (7). Survivin is extensively expressed in the majority of cancer types including lung, breast, liver, colon, pancreatic, and head and neck cancer, but is not expressed or is expressed at a substantially lower level in normal tissue $(8,9)$, which makes it a potential target for anticancer therapy (10). Mounting evidence suggested that survivin expression is associated with drug resistance in cancer cells and a poor clinical outcome in patients with cancer (11-14). In addition, survivin inhibition by siRNA or by survivin antisense oligonucleotides (SAO) has been demonstrated to sensitize tumors to cytotoxic drugs inculding cisplatin in vitro and in vivo (11-16).

Sepantronium bromide (YM155), a novel small molecule inhibitor of survivin, was obtained from high throughput screening using a survivin promoter luciferase assay (17). YM155 demonstrates potent antitumor activities against a various types of cancer, such as non-small cell lung cancer, nonHodgkin lymphoma, melanoma and prostate cancer (17-22). Of note, phase I and II trials with YM155 have shown its safety and tolerability in patients with advanced refractory non-small cell lung carcinoma (23) or unresectable melanoma (24). Recent findings have shown that YM155 enhanced the antitumor activity of cytotoxic agents in several cell lines (25).

Thus, we hypothesized that inhibition of survivin expression by YM155 in hepatoblastoma cells may enhance the therapeutic efficacy of CDDP by inhibiting the acquisition of chemoresistance in cancer cells. Therefore, in this study, we assessed the therapeutic potential of YM155 alone or in 
combination with cisplatin in preclinical hepatoblastoma models in vitro and in vivo.

\section{Materials and methods}

Tissue and cell lines. Thirty patients were treated at the Department of Pediatric Surgery, The First Hospital of Jilin University (Changchun, China) between August 2011 and August 2014. The present study was approved by the Research Ethics Committee of Jilin University (Changchun, China). Written informed consent was obtained from all of the patients. Fresh hepatoblastoma and corresponding normal liver tissues $5 \mathrm{~cm}$ away from the edge of the hepatoblastoma tissue were taken from the patients with hepatoblastoma who had not received any chemotherapy or radiotherapy prior to surgery. The samples were then snap-frozen in liquid nitrogen immediately after resection and kept at $-80^{\circ} \mathrm{C}$.

Human HepG2 and HuH6 hepatoblastoma cell lines, purchased from the Cell Bank of Type Culture Collection of Chinese Academy of Sciences, Shanghai Institute of Cell Biology (Shanghai, China) were cultured in DMEM and RPMI-1640 medium, respectively, with $10 \%$ fetal bovine serum (FBS; Gibco-Life Technologies, Carlsbad, CA, USA), $100 \mathrm{IU} / \mathrm{ml}$ penicillin and $100 \mu \mathrm{g} / \mathrm{ml}$ streptomycin at $37^{\circ} \mathrm{C}$ in a humidified atmosphere containing $5 \% \mathrm{CO}_{2}$. Primary human fibroblasts were isolated from the skin biopsies of healthy adult donors, and were cultured as adherent monolayers in DMEM containing $15 \%$ FBS, $1 \%$ glutamine and $1 \%$ penicillin/streptomycin. The cells were subcultured every 2 or 3 days.

Reagents and antibodies. YM155 was obtained from Selleck Chemicals (Houston, TX, USA). Cisplatin was purchased from Sigma-Aldrich Co. (St. Louis, MO, USA). The antibodies used for the western blot analysis were: mouse anti-human survivin, and mouse anti-human GAPDH, mouse anti-human cleaved caspase- 3 , mouse anti-human cleaved caspase- 8 and mouse anti-human RARP monoclonal antibodies were purchased from Cell Signaling Technology (Beverly, MA, USA).

Quantification by quantitative polymerase chain reaction $(q P C R)$. Total RNA of liver tissue and cultured cells was extracted using TRIzol reagent (Invitrogen, Carlsbad, CA, USA) according to the manufacturer's instructions and quantified with the NanoDrop 2000 (Thermo Fisher Scientific, Tokyo, Japan). Reverse transcription of $1 \mu \mathrm{g}$ of total RNA was performed using oligo(dT) primers (Invitrogen) and reverse transcriptase (Toyobo, Co., Osaka, Japan) to obtain complementary deoxyribonucleic acid (cDNA). qPCR assays were carried out using SYBR-Green Real-Time PCR Master Mix (Toyobo) and amplification equipment ABI PRISM 7900HT (Applied Biosystems, Foster City, CA, USA). GADPH was used as the endogenous control for quantifying mRNA levels. The survivin and GAPDH primer sequences were designed as described by Zhang et al (26). The $2^{-\Delta \Delta \mathrm{CT}}$ method was used to calculate the relative abundance of target gene expression generated by Rotor-Gene Real-Time Analysis software 6.1.81 (Qiagen, Hilden, Germany).

Cell proliferation assay. To determine the inhibitory effect of YM155 alone or in combination on cell proliferation, a 3-(4,5-dimethylthiazol-2-yl)-2,5-diphenyltetrazolium bromide (MTT) assay was performed. Cells $\left(1 \times 10^{4} /\right.$ well) were plated in 96-well plates and then cultured in medium with or without various concentrations of YM155 and/or CDDP alone or in combination. Control cultures received 0.1\% DMSO. Three days after the treatment, the percentage of viable cells in each well was examined by MTT assay (Sigma Chemical), using the SpectraMax Plus spectrophotometer (Molecular Devices, Sunnyvale, CA, USA) at a wavelength of $570 \mathrm{~nm}$ by a spectrophotometer. The percentage of viable cells for each treatment group was calculated by adjusting the untreated control group to $100 \%$.

Colony formation assays. Cells were seeded in 6-well plates at a density of $1 \times 10^{3}$ cells and were exposed to their respective half maximal inhibitory concentration $\left(\mathrm{IC}_{50}\right)$ values of YM155, CDDP or both for $48 \mathrm{~h}$. The cells were then washed with drugs-free medium and allowed to grow for 14 days in drugs-free conditions. The percentage colony formation was calculated by adjusting untreated cells to $100 \%$. All the experiments were performed in triplicate.

Apoptosis assay. Apoptosis was performed using an Annexin V binding assay (ApoAlert Annexin V-FITC apoptosis kit; Clontech Laboratories, Inc., Mountain View, CA, USA). Briefly, the cells were treated with their respective $\mathrm{IC}_{50}$ values of YM155, CDDP or both for $48 \mathrm{~h}$. Then cells were washed with PBS twice and resuspended in $100 \mu 1$ binding buffer (Sigma). Subsequently, $5 \mu$ l Annexin V FITC was added and incubated for $15 \mathrm{~min}$ in the dark at room temperature. The cells were analyzed with a Becton-Dickinson FACScan flow cytometer using the CellsQuest program 2.6 (Becton-Dickinson).

In addition, the expression of cleaved caspase-3, cleaved caspase- 8 and cleaved PARP was determined by western blot analysis $24 \mathrm{~h}$ after treatment with their respective $\mathrm{IC}_{50}$ values of YM155, CDDP or both.

Western blot analysis. Protein was extracted from tissues or cells using lysis buffer (RIPA Buffer; Sigma Chemical). Protein concentrations of lysates were determined using a BCA protein assay reagent kit (Tiangen Biotech, Beijing, China). Equal amounts of total protein $(20 \mu \mathrm{g})$ were resolved by $10-12 \%$ SDS-PAGE and transferred to a polyvinylidene fluoride membrane. After blocking at room temperature with with $5 \%$ dry milk in TBS-T, each membrane was incubated overnight at $4^{\circ} \mathrm{C}$ with the primary antibodies. After washing with TBS-T twice, the membranes were incubated with horseradish peroxidase-conjugated secondary antibody for $1 \mathrm{~h}$ at room temperature. Proteins of interest were visualized by enhanced chemiluminescence using ECL Prime (GE Healthcare, Buckinghamshire, UK). Blots were stripped and reprobed with anti-GAPDH to control for loading variations. Quantity One software (Bio-Rad, Hercules, CA, USA) was used for quantification of the protein bands.

Xenograft model. To assess the effect of CDDP and/or YM155 on tumor growth in vivo, we used a nude mouse tumor xenograft model. Six-week-old male nude mice (BALB/c nu/nu) were purchased from the Experiments Animal Center of Changchun Biological Institute (Changchun, China). HepG2 

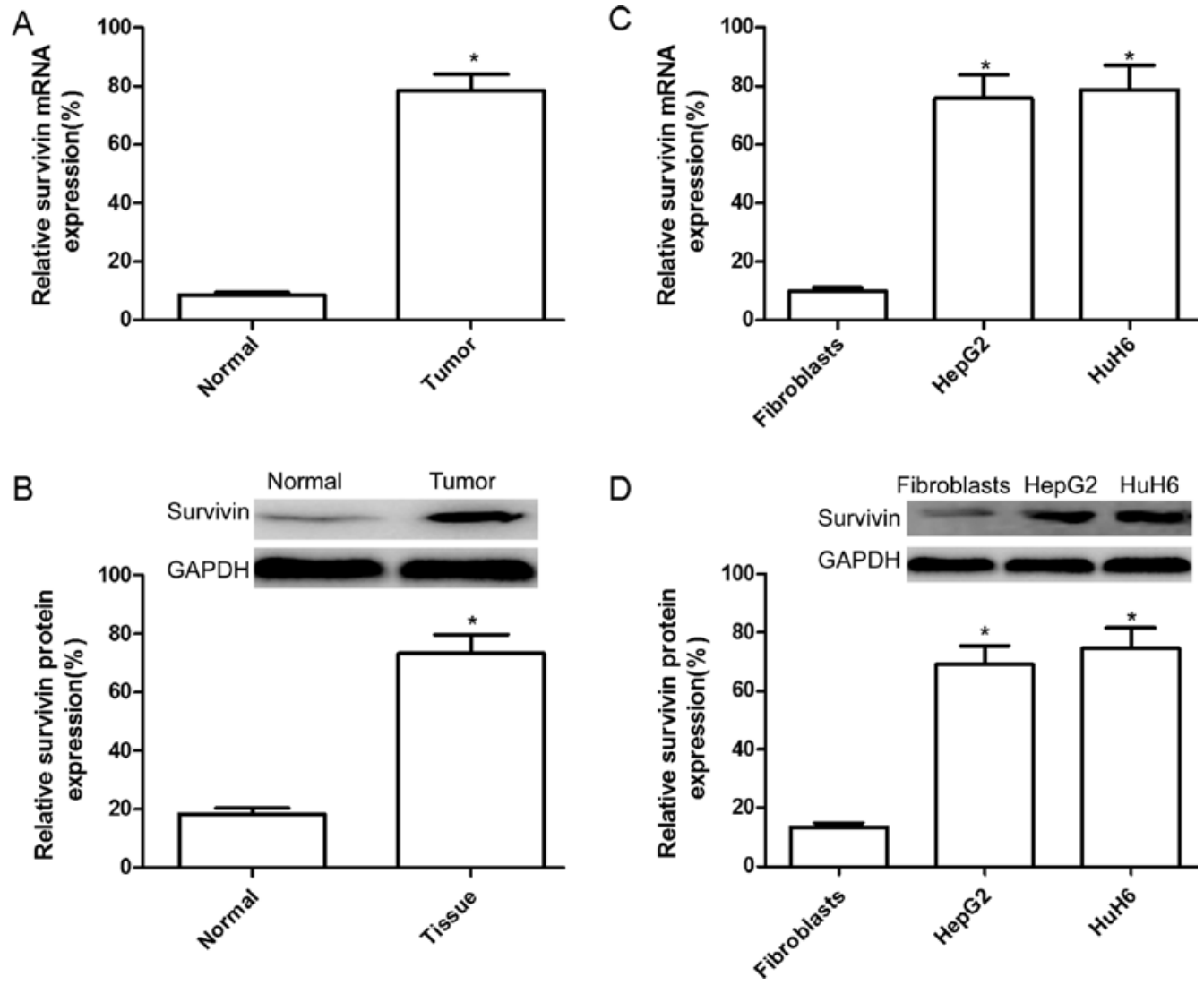

Figure 1. Survivin expression is upregulated in hepatoblastoma cell lines and patient's primary tumor samples. (A) Quantitative RT-PCR (RT-qPCR) results of relative mRNA expression level of survivin in 30 cases of hepatoblastoma tumor tissue and adjacent normal tissue samples. The mRNA expression level of GAPDH was quantified as an internal control. ${ }^{~} \mathrm{P}<0.05$ vs. normal tissue. (B) Western blot results of survivin protein expression in hepatoblastoma tumor tissue and matched adjacent normal tissues. " $\mathrm{P}<0.05$ vs. normal tissue. (C) RT-qPCR results of relative mRNA expression level of survivin in hepatoblastoma cell lines (HepG2 and HuH6) and normal fibroblasts. ${ }^{*} \mathrm{P}<0.05$ vs. fibroblasts. (D) Western blot results of survivin protein expression level of survivin in hepatoblastoma cell lines (HepG2 and HuH6) and normal fibroblasts are shown. ${ }^{*} \mathrm{P}<0.05$ vs. fibroblasts.

cells $\left(2 \times 10^{6}\right)$ were injected into the flanks of the mice and allowed to reach a tumor volume of $>100 \mathrm{~mm}^{3}$ in tumor volume (length $\mathrm{x}$ width2)/2. The mice were randomly divided into four groups $(\mathrm{n}=10 \mathrm{mice} / \mathrm{group})$. The control group received $1 \%$ polysorbate resuspended in deionized water. The remaining three groups were treated with YM155 $(10 \mathrm{mg} / \mathrm{kg}$ body weight), CDDP ( $3 \mathrm{mg} / \mathrm{kg}$ body weight), or YM155 plus CDDP (3 and $1 \mathrm{mg} / \mathrm{kg}$ body weight, respectively) intraperitoneally for three days over 4 weeks. The tumor volume was measured on day 7, 14, 21 and 28 of the treatment. On day 28, the animals were euthanized using chloroform, tumor tissues were resected and volume and weight were measured. The survivin expression levels of tumor tissues were analyzed by western blot analysis. The study was approved by the Jilin University Animal Care and Experimentation Committee (Changchun, China).

Statistical analysis. Data were presented as mean \pm SD (standard deviation). Comparisons between two groups were analyzed by the Student's paired t-test. A comparison of $\geq 2$ groups was performed using one-way ANOVA followed by a Tukey's post hoc test. Statistical analyses were performed using GraphPad Prism, version 5 (GraphPad Software, Inc., San Diego, CA, USA) and SPSS software (version 16.0; SPSS Inc., Chicago, IL, USA). P $<0.05$ was considered statistically significant.

\section{Results}

Expression of survivin is upregulated in hepatoblastoma tissues and cells. To identify the potential roles of survivin in the occurrence and development of hepatoblastoma, we detected survivin expression levels in tumor tissues and adjacent normal tissues from 30 patients with hepatoblastoma by reverse-transcriptase quantitative PCR (RT-qPCR) and western blot analysis. Results of RT-qPCR showed that the mRNA expression level of surivivn in hepatoblastoma tumor tissues was significantly increased compared to matched adjacent normal tissues (Fig. 1A; $\mathrm{P}<0.05$ ). In addition, elevated levels of survivin protein were found in hepatoblastoma tumor tissues compared with the paired adjacent normal tissues by western blot analysis (Fig. 1B).

The expression levels of survivin in human HepG2 and HuH6 hepatoblastoma cell lines, and normal fibroblasts were detected by RT-qPCR and western blot analysis. It was found that the expression of survivin on the mRNA (Fig. 1C) and protein (Fig. 1D) levels were clearly upregulated in HepG2 and HuH6 cells compared to the normal fibroblasts $(\mathrm{P}<0.05)$.

YM155 inhibits survivin expression in hepatoblastoma cells. YM155 (Fig. 2A), a novel small molecule inhibitor of survivin, has been shown to inhibit survivin expression in various types of cancer (17-22). We examined whether YM155 was effective 
A<smiles>COCCn1c2c([n+](Cc3cnccn3)c1C)C(=O)c1ccccc1C2=O</smiles>

B

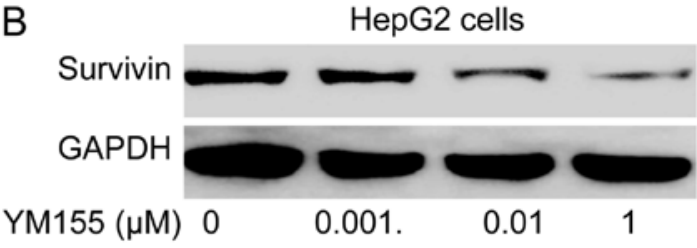

C

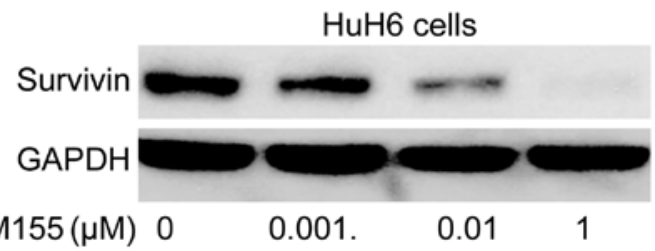

Figure 2. YM155 inhibits survivin expression of hepatoblastoma cell lines in a dose-dependent manner. (A) Chemical structure of YM155. (B) HepG2 and (C) HuH6 cells were treated with different doses of YM155 $(0,0.001,0.01$ and $1 \mu \mathrm{M})$ for $24 \mathrm{~h}$, and survivin expression was examined by western blot analysis. GAPDH was used as a control.

A

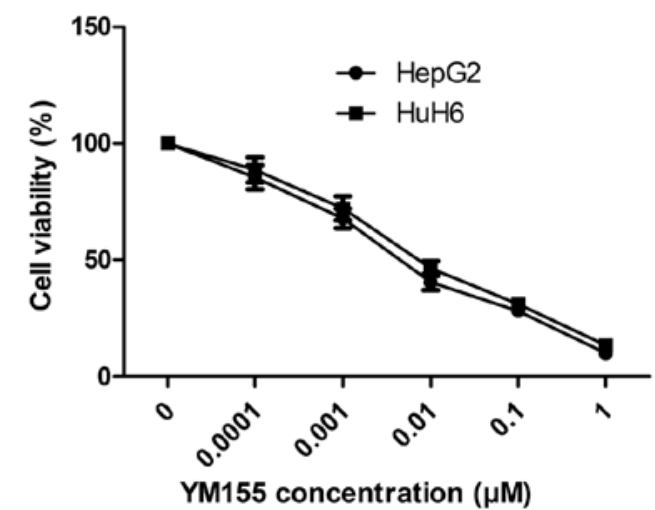

C

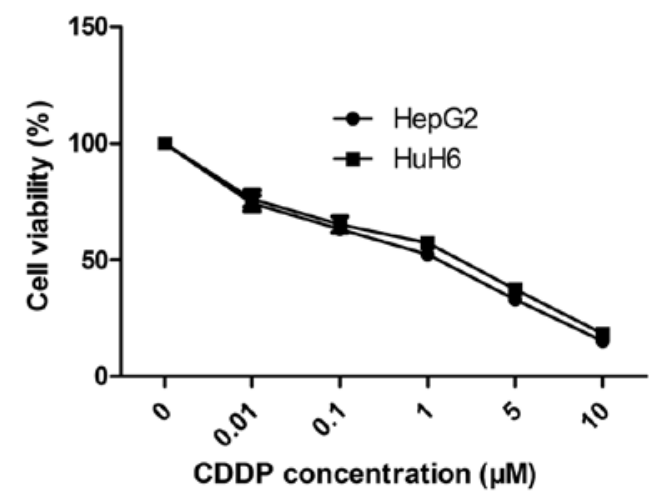

B


Figure 3. Effect of YM155 and cisplatin (CDDP) alone or combination on cell proliferation and cell colony formation in hepatoblastoma cell lines. Cell viability assay of HepG2 and HuH6 were performed $72 \mathrm{~h}$ after treated with various concentrations of (A) YM155 (0-1 $\mu \mathrm{M})$, (B) CDDP (0-10 $\mu \mathrm{M})$, or (C) both $(0.001 \mu \mathrm{M}$ YM155 plus 0-10 $\mu \mathrm{M} \mathrm{CDDP})$ and $\mathrm{IC}_{50}$ were calculated. (D) Clone formation of hepatoblastoma cells was counted after treated with their IC $\mathrm{C}_{50}$ concentration of YM155 and CDDP individually and their combinations. ${ }^{*} \mathrm{P}<0.05$ vs. control, ${ }^{*} \mathrm{P}<0.05$ vs. CDDP alone.

in inhibiting survivin expression on protein level in hepatoblastoma cell lines by western blot analysis $24 \mathrm{~h}$ following treatment with different doses of YM155 $(0,0.001,0.01$ and $1 \mu \mathrm{M})$. It was found that YM155 significantly downregulated survivin protein expression in HepG2 and $\mathrm{HuH} 6$ cells in a dose-dependent manner (Fig. 2B and C).
YM155 and CDDP alone or in combination inhibit cell proliferation and colony formation in hepatoblastoma cell lines. To assess the effect of YM155 and CDDP alone or in combination on the cell viability of hepatoblastoma cells in vitro, HepG2 and HuH6 cells were treated with different drug concentrations of YM155 $(0-1 \mu \mathrm{M})$, CDDP $(0-10 \mu \mathrm{M})$, 


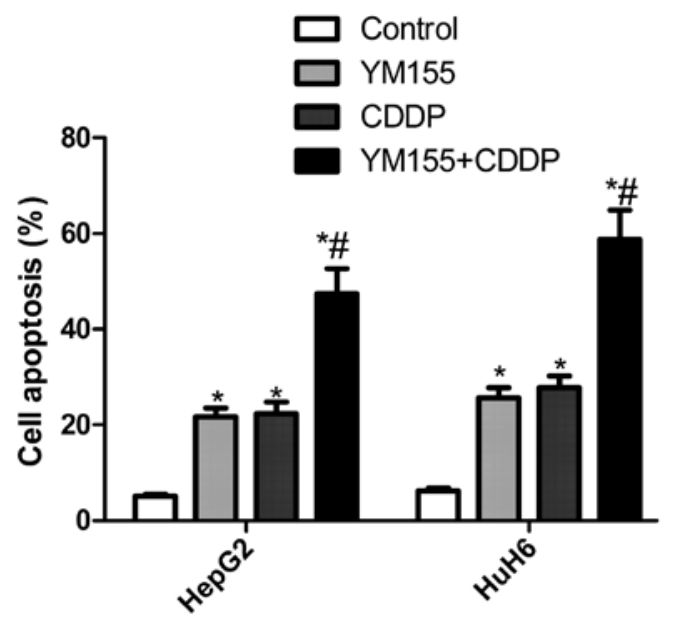

Figure 4. Effect of YM155 and CDDP alone or in combination on cell apoptosis in hepatoblastoma cell lines. HepG2 and HuH6 cells were treated with YM155 and CDDP alone or in combination at their $\mathrm{IC}_{50}$ concentrations. After treatment for $48 \mathrm{~h}$, cell apoptosis was determined by flow cytometry. ${ }^{*} \mathrm{P}<0.05$ vs. control, ${ }^{\#} \mathrm{P}<0.05$ vs. CDDP alone.

or both (0.001 $\mu \mathrm{M}$ YM155 plus $0-10 \mu \mathrm{M}$ CDDP) for $72 \mathrm{~h}$, respectively, and an MTT assay were performed. It was found that YM155 inhibited the cell proliferation of the HepG2 and HuH6 in a dose-dependent manner. The $\mathrm{IC}_{50}$ values of YM155 were 64 and $76 \mathrm{nM}$ for HepG2 and HuH6 cell line, respectively (Fig. 3A). CDDP alone also reduced cell proliferation in hepatoblastoma cell lines in a dose-dependent manner with an $\mathrm{IC}_{50}$ of $5.4 \mu \mathrm{M}$ in HepG2 cells and an $\mathrm{IC}_{50}$ of $6.1 \mu \mathrm{M}$ in HuH6 cells (Fig. 3B). Combination treatment (0-10 $\mu \mathrm{M}$ CDDP in the presence of $0.001 \mu \mathrm{M}$ YM155) reduced cell proliferation in hepatoblastoma cell lines in a dose-dependent manner with an $\mathrm{IC}_{50}$ of $2.1 \mu \mathrm{M}$ in HepG2 cells and an $\mathrm{IC}_{50}$ of $2.4 \mu \mathrm{M}$ in HuH6 cells (Fig. 3C), indicating that YM155 enhanced the sensitivity of CDDP to hepatoblastoma cell lines. Based on the results, we selected respective $\mathrm{IC}_{50}$ values of drugs for subsequent treatments throughout the study.

The effects of YM155 and CDDP alone or in combination on the cell colony formation of hepatoblastoma cell lines were also determined. As shown Fig. 3D, the colony formation number of the HepG2 and HuH6 hepatoblastoma cells was significantly reduced in the YM155 and CDDP alone or combination groups. Combination treatment with YM155 and CDDP resulted in an even greater percentage of reduction.

YM155 and CDDP alone or in combination induce cell apoptosis in hepatoblastoma cell lines. We examined the in vitro effect of YM155 and CDDP alone or in combination on cell apoptosis in hepatoblastoma cell lines. The results showed that exposure to YM155 increased the apoptotic cells by $21.7 \%$ in HepG2 cells and $25.6 \%$ in HuH6 cells (Fig. 4), and exposure to CDDP increased the apoptotic cells by $22.4 \%$ in HepG2 cells and $27.8 \%$ in HuH6 cells. By contrast, the combination of YM155 and CDDP showed that the apoptotic cells were increased by 47.3 and $58.8 \%$ in HepG2 and HuH6 cells, respectively, which was greater than either agent alone (Fig. 4).

YM155 and CDDP alone or in combination induces caspase activation. To assess the ability of YM155 and CDDP alone or in combination to activate caspase -3 and -8 activity, we determined cleaved caspase -3 and -8 expression by western blot analysis following treatment with YM155 and CDDP alone or in combination. We found that cleaved caspase- 3 and -8 expression was significantly upregualted in the YM155 and CDDP alone or combination treatment groups as compared to the controls group (Fig. 5). Combination treatment significantly increased the cleaved caspase-3 and -8 expression levels as compared to YM155 or CDDP treatment alone (Fig. 5). Furthermore, we determined the cleaved PARP expression in the hepatoblastoma cell lines following treatment with YM155 and CDDP alone or in combination. Western blot analysis revealed that the expression of cleaved PARP was upregulated in cells treated with YM155 and CDDP alone or in combination as compared to the control (Fig. 5). Combination treatment led to maximal addition for cleaved PARP expression.

Antitumor effect of YM155 combined with CDDP in a xenograft model. The in vitro data suggested that YM155 and CDDP alone or in combination significantly inhibited cancer cell growth. To validate our in vitro results further, we carried out YM155 and CDDP combination treatment experiment in a HepG2 xenograft mouse model. Tumors grown in nude mice were respectively treated with YM155, CDDP or both for four weeks. The tumors were then extracted and it was found that they were significantly smaller in the YM155 and CDDP alone

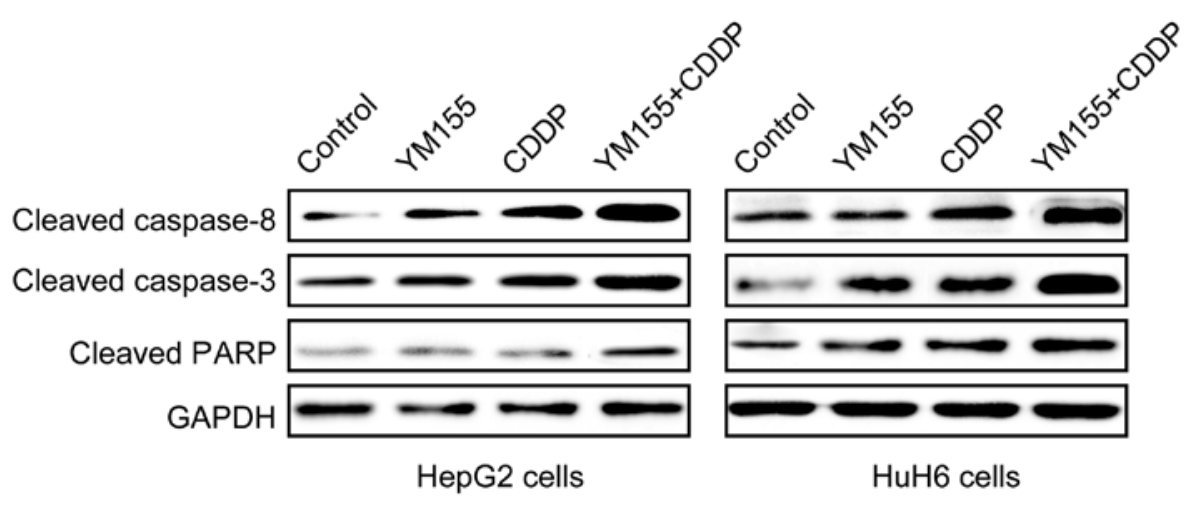

Figure 5. Effect of YM155 and CDDP alone or in combination on expression of cleaved caspase-3, caspase-8 and RARP in hepatoblastoma cell lines. Cells were treated with YM155 and CDDP alone or combination for $24 \mathrm{~h}$, and the expression of cleaved caspase-3, cleaved caspase-8 and cleaved RARP was detected by western blot analysis. GAPDH was used as loading control. 
A

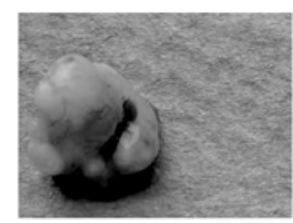

Control

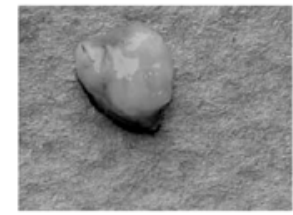

CDDP

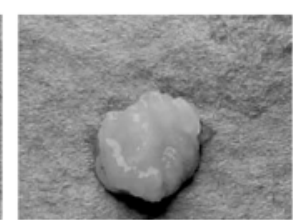

YM155



YM155+CDDP



B

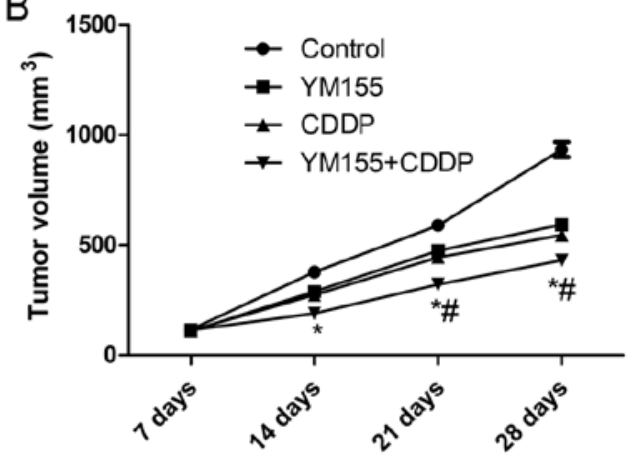

D

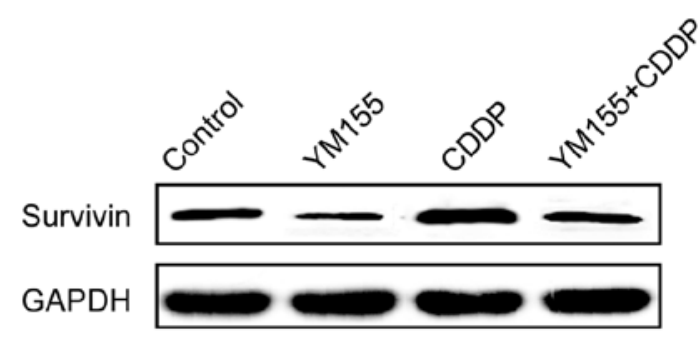

Figure 6. Antitumor activity of YM155 and CDDP alone or combination in BALB/c mice bearing HepG2 cells. (A) Representative photomicrographs of tumors from untreated (Control), YM155, CDDP and combination group. (B) Relative change of tumor volume curves for HepG2 tumors treated with YM155 and CDDP alone or combination. (C) Tumor weight from untreated (control), YM155, CDDP and combination group. (D) Western blot analyses for survivin level in tumor tissues. ${ }^{*} \mathrm{P}<0.05$ vs. control, ${ }^{*} \mathrm{P}<0.05$ vs. CDDP alone.

or in combination groups than that of the control group. The combination treatment group led to marked inhibition of tumor growth as compared to the monotherapy groups (Fig. 6A). Additionally, tumor volume (Fig. 6B) and tumor weight (Fig. 6C) following treatment with YM155 and CDDP alone or in combination were significantly reduced as compared to the control group $(\mathrm{P}<0.05$; Fig. $6 \mathrm{~B}$ and $\mathrm{C})$. Treatment with the combination of YM155 and CDDP resulted in marked inhibitoin of tumor growth as compared to monotherapy $(\mathrm{P}<0.05$; Fig. 6B and $\mathrm{C}$ ). We also determined survivin expression in xenograft tumors by western blot analysis. The results revealed that survivin expression was upregulated in the CDDP treatment group as compared to the remaining groups, while survivin expression was downregulated in the YM155 treatment group as compared to the remaining groups (Fig. 6D). Of note, survivin expression had no significant change in the combination group as compared to the control group (Fig. 6D). These results showed that YM155 in combination with CDDP treatment markedly suppressed hepatoblastoma tumorigenicity in nude mice.

\section{Discussion}

In the present study, we examined the validity of targeting survivin and the therapeutic potential of YM155 alone and in combination with CDDP in vitro and in vivo. We found that survivin expression was increased in hepatoblastoma tumor tissues and hepatoblastoma cell lines. In an in vitro combination study, YM155 synergistically enhanced the antitumor activity of CDDP in hepatoblastoma cell lines by inhibiting hepatoblastoma cell proliferation and colony formation, and inducing cell apoptosis. In addition, YM155 concomitantly combined with CDDP led to greater tumor reduction than single YM155 or CDDP treatment in established xenograft models. These findings suggest that YM155 in combination with CDDP is a promising candidate for hepatoblastoma therapy.

Survivin, the smallest member of the inhibitor of apoptosis (IAP) family, consist of 142 amino acids. It has been reported that it is strongly expressed in children with various types of malignancies, including neuroblastoma (27), soft tissue sarcoma and bone sarcoma (28). It was recently shown that the survivin expression was significantly increased in hepatoblastoma tumor tissues and HuH6 cells after chemotherapy (29). The present study has shown that survivin expression was significantly upregulated in hepatoblastoma tumor tissue and HepG2 and HuH6 hepatoblastoma cell lines as compared to adjacent normal live tissue or normal fibroblasts. Mounting evidence has shown that survivin promotes cell proliferation by facilitating accurate mitotic progression and inhibits cell apoptosis by inhibiting caspase activation $(30,31)$. Thus, survivin is an attractive target for treating hepatoblastoma using a small molecule antagonist or immunotherapy to inhibit survivin expression. YM155, a novel small-molecule survivin suppressant, has demonstrated potent anticancer activity against a broad spectrum of human cancer cell lines and various human-derived tumor xenograft mouse models 
by altering intratumoral survivin expression levels and a subsequent direct apoptosis induction (19). To the best of our knowledge, our results are the first to show that YM155 alone or in combination with CDDP may effectively suppress tumor growth of hepatoblastoma in vitro and in vivo by inhibiting survivin expression.

It is well known that combinatorial chemotherapy potentially achieves an improved response rate as compared to that of monotherapy since combination treatment mainly depends on the synergism between different therapeutic agents with different mechanisms of action to increase efficacy, while maintaining a favorable side-effect and decreasing overlapping toxicity. Accumulating evidence suggests YM155 alone or in combination with other types of chemotherapy suppresses tumor growth in several cancers in vitro or in vivo. For example, Nakahara et al (32) have reported that tumor regression induced by YM155 in combination with docetaxel was accompanied by decreased intratumoral survivin, resulting in an apoptotic rate as compared to either treatment alone. Kaneko et al (33) have shown that YM155 enhances the antitumor activity of bendamustine against DLBCL models through inhibition of DNA damage responses as well as survivin-mediated cytoprotection at the G2-M phase. Liang et al (34) showed that the concomitant combination of YM155 with cisplatin induced more intense apoptosis in SH-SY5Y human neuroblastomas cells compared with monotherapy in vitro, and that this combination shows greater efficacy than either agent alone in mouse xenograft models. Kumar et al (25) found that YM155 significantly enhanced the antitumor and antiangiogenic effects of CDDP in head and neck squamous cell carcinoma, with no added systemic toxicity. In the present study, we found that YM155 could enhance sensitivity to the antitumor effect of CDDP in human HepG2 and HuH6 hepatoblastoma cell lines and that the combination treatment of YM155 and CDDP promoted tumor regression of hepatoblastoma in vitro and in vivo, suggesting a potentially novel strategy to employing YM155 to overcome resistance in tumor cells, thereby enhancing the effectiveness of CDDP in hepatoblastoma.

Survivin may block apoptosis by inhibiting caspase-3 and -7 activities directly (35). Previous findings have shown that inhibiting survivin by YM155 may increase cleaved caspase-3 expression levels, indicating activation of caspase-3, resulting in cell apoptosis in human malignant cells (36). In addition, it has been shown that caspases- 8 plays an important role in the apoptotic cell death via the extrinsic apoptosis signaling cascade (37). Recent results have shown that YM155 induced apoptosis in human leukemia cells concomitant with the activation of caspase-3 and -8 (38). Consistent with these results, the present study has shown that YM155 alone or in combination with CDDP significantly induced cell apoptosis by increasing cleaved caspase- 3 and -8 expression.

In conclusion, the present study has shown that survivin expression was upregulated in hepatoblastoma tumor tissues and cell lines, and that YM155 significantly enhanced the therapeutic efficacy of cisplatin treatment. Additionally, YM155 in combination with CDDP synergistically decreased hepatoblastoma cell proliferation and formation, induced cell apoptosis in vitro, and suppressed the tumor growth of hepatoblastoma in a nude mouse model. These findings suggest that the combination of YM155 and CDDP is a promising drug candidate for the treatment of hepatoblastoma.

\section{Acknowledgements}

The present study was supported by the Innovation Platform Construction Approach and Strategy on the Single-Disease Clinical Pathway of Jilin Province (20120626), and the Fundamental Public Service System Construction Approach and Strategy on Single-Disease Clinical Pathway of Changchun City (2012117).

\section{References}

1. Stocker JT: Hepatoblastoma. Semin Diagn Pathol 11: 136-143, 1994.

2. Schoofs G, Braeye L, Vanheste R, Verswijvel G, DebiecRychter M and Sciot R: Hepatic rhabdomyosarcoma in an adult: A rare primary malignant liver tumor. Case report and literature review. Acta Gastroenterol Belg 74: 576-581, 2011.

3. Czauderna P, Lopez-Terrada D, Hiyama E, Haberle B, Malogolowkin MH and Meyers RL: Hepatoblastoma state of the art: Pathology, genetics, risk stratification, and chemotherapy. Curr Opin Pediatr 26: 19-28, 2014.

4. Davies JQ, de la Hall PM, Kaschula RO, Sinclair-Smith CC, Hartley P, Rode H and Millar AJ: Hepatoblastoma - evolution of management and outcome and significance of histology of the resected tumor. A 31-year experience with 40 cases. J Pediatr Surg 39: 1321-1327, 2004

5. Zhang YT, Feng LH, Zhong XD, Wang LZ and Chang J: Single-agent cisplatin treatment of children with high-risk hepatoblastoma. J Pediatr Hematol Oncol 36: 271-275, 2014.

6. Qayed M and Katzenstein HM: Dose-intensive cisplatin for hepatoblastoma: Have you heard? Lancet Oncol 14: 791-792, 2013.

7. Colnaghi R, Connell CM, Barrett RM and Wheatley SP: Separating the anti-apoptotic and mitotic roles of survivin. J Biol Chem 281: 33450-33456, 2006.

8. Altieri DC: Validating survivin as a cancer therapeutic target. Nat Rev Cancer 3: 46-54, 2003.

9. Duffy MJ, O'Donovan N, Brennan DJ, Gallagher WM and Ryan BM: Survivin: A promising tumor biomarker. Cancer Lett 249: 49-60, 2007.

10. Ambrosini G, Adida C and Altieri DC: A novel anti-apoptosis gene, survivin, expressed in cancer and lymphoma. Nat Med 3: 917-921, 1997.

11. Asechi H, Hatano E, Nitta T, Tada M, Iwaisako K, Tamaki N, Nagata H, Narita M, Yanagida A, Ikai I, et al: Resistance to cisplatin-induced apoptosis via PI3K-dependent survivin expression in a rat hepatoma cell line. Int J Oncol 37: 89-96, 2010.

12. Chandele A, Prasad V, Jagtap JC, Shukla R and Shastry PR: Upregulation of survivin in $\mathrm{G} 2 / \mathrm{M}$ cells and inhibition of caspase 9 activity enhances resistance in staurosporine-induced apoptosis. Neoplasia 6: 29-40, 2004.

13. Tirro E, Consoli ML, Massimino M, Manzella L, Frasca F, Sciacca L, Vicari L, Stassi G, Messina L, Messina A, et al: Altered expression of c-IAP1, survivin, and Smac contributes to chemotherapy resistance in thyroid cancer cells. Cancer Res 66: 4263-4272, 2006.

14. Zaffaroni N, Pennati M, Colella G, Perego P, Supino R, Gatti L, Pilotti S, Zunino F and Daidone MG: Expression of the antiapoptotic gene survivin correlates with taxol resistance in human ovarian cancer. Cell Mol Life Sci 59: 1406-1412, 2002.

15. Kunze D, Erdmann K, Froehner M, Wirth MP and Fuessel S: siRNA-mediated inhibition of antiapoptotic genes enhances chemotherapy efficacy in bladder cancer cells. Anticancer Res 32: 4313-4318, 2012.

16. Hayashi N, Asano K, Suzuki H, Yamamoto T, Tanigawa N, Egawa $\mathrm{S}$ and Manome Y: Adenoviral infection of survivin antisense sensitizes prostate cancer cells to etoposide in vivo. Prostate 65: 10-19, 2005.

17. Nakahara T, Kita A, Yamanaka K, Mori M, Amino N, Takeuchi M, Tominaga F, Hatakeyama S, Kinoyama I, Matsuhisa A, et al: YM155, a novel small-molecule survivin suppressant, induces regression of established human hormone-refractory prostate tumor xenografts. Cancer Res 67: 8014-8021, 2007. 
18. Kawano H, Shakushiro K, Nakata M, Kita A, Maeda A, Watanabe S, Sako K and Oku N: Antitumor efficacy and biodistribution of liposomal sepantronium bromide (YM155), a novel small-molecule survivin suppressant. Eur J Pharm Biopharm 88: 283-289, 2014.

19. Nakahara T, Kita A, Yamanaka K, Mori M, Amino N, Takeuchi M, Tominaga F, Kinoyama I, Matsuhisa A, Kudou M, et al: Broad spectrum and potent antitumor activities of YM155, a novel small-molecule survivin suppressant, in a wide variety of human cancer cell lines and xenograft models. Cancer Sci 102: 614-621, 2011

20. Minematsu T, Iwai M, Sugimoto K, Shirai N, Nakahara T, Usui $\mathrm{T}$ and Kamimura $\mathrm{H}$ : Carrier-mediated uptake of 1-(2-methoxyethyl)-2-methyl-4,9-dioxo-3-(pyrazin-2-ylmethyl)4,9-dihydro-1H-naphtho[2,3-d]imidazolium bromide (YM155 monobromide), a novel small-molecule survivin suppressant, into human solid tumor and lymphoma cells. Drug Metab Dispos 37: 619-628, 2009

21. Kita A, Nakahara T, Yamanaka K, Nakano K, Nakata M, Mori M, Kaneko N, Koutoku H, Izumisawa N and Sasamata M: Antitumor effects of YM155, a novel survivin suppressant, against human aggressive non-Hodgkin lymphoma. Leuk Res 35: 787-792, 2011.

22. Tolcher AW, Quinn DI, Ferrari A, Ahmann F, Giaccone G, Drake T, Keating A and de Bono JS: A phase II study of YM155, a novel small-molecule suppressor of survivin, in castrationresistant taxane-pretreated prostate cancer. Ann Oncol 23: 968-973, 2012.

23. Giaccone G, Zatloukal P, Roubec J, Floor K, Musil J, Kuta M, van Klaveren RJ, Chaudhary S, Gunther A and Shamsili S: Multicenter phase II trial of YM155, a small-molecule suppressor of survivin, in patients with advanced, refractory, non-small-cell lung cancer. J Clin Oncol 27: 4481-4486, 2009.

24. Lewis KD, Samlowski W, Ward J, Catlett J, Cranmer L, Kirkwood J, Lawson D, Whitman E and Gonzalez R: A multicenter phase II evaluation of the small molecule survivin suppressor YM155 in patients with unresectable stage III or IV melanoma. Invest New Drugs 29: 161-166, 2011.

25. Kumar B, Yadav A, Lang JC, Cipolla MJ, Schmitt AC, Arradaza N, Teknos TN and Kumar P: YM155 reverses cisplatin resistance in head and neck cancer by decreasing cytoplasmic survivin levels. Mol Cancer Ther 11: 1988-1998, 2012.

26. Zhang K, Li Y, Liu W, Gao X and Zhang K: Silencing survivin expression inhibits the tumor growth of non-small-cell lung cancer cells in vitro and in vivo. Mol Med Rep 11: 639-644, 2015.
27. Ito R, Asami S, Motohashi S, Ootsuka S, Yamaguchi Y, Chin M, Shichino H, Yoshida Y, Nemoto N, Mugishima H, et al: Significance of survivin mRNA expression in prognosis of neuroblastoma. Biol Pharm Bull 28: 565-568, 2005.

28. Kappler M, Köhler T, Kampf C, Diestelkötter P, Würl P, Schmitz M, Bartel F, Lautenschläger C, Rieber EP, Schmidt H, et al: Increased survivin transcript levels: an independent negative predictor of survival in soft tissue sarcoma patients. Int J Cancer 95: 360-363, 2001

29. Uehara S, Oue T, Kawatsu M, Nara K and Fukuzawa M: Increased expression of survivin in hepatoblastoma after chemotherapy. Eur J Pediatr Surg 23: 400-404, 2013.

30. Mita AC, Mita MM, Nawrocki ST and Giles FJ: Survivin: Key regulator of mitosis and apoptosis and novel target for cancer therapeutics. Clin Cancer Res 14: 5000-5005, 2008.

31. Ryan BM, O'Donovan N and Duffy MJ: Survivin: A new target for anti-cancer therapy. Cancer Treat Rev 35: 553-562, 2009.

32. Nakahara T, Yamanaka K, Hatakeyama S, Kita A, Takeuchi M, Kinoyama I, Matsuhisa A, Nakano K, Shishido T, Koutoku H, et al: YM155, a novel survivin suppressant, enhances taxaneinduced apoptosis and tumor regression in a human Calu 6 lung cancer xenograft model. Anticancer Drugs 22: 454-462, 2011.

33. Kaneko N, Mitsuoka K, Amino N, Yamanaka K, Kita A, Mori M, Miyoshi S and Kuromitsu S: Combination of YM155, a survivin suppressant, with bendamustine and rituximab: A new combination therapy to treat relapsed/refractory diffuse large B-cell lymphoma. Clin Cancer Res 20: 1814-1822, 2014.

34. Liang H, Zhang L, Xu R and Ju XL: Silencing of survivin using YM155 induces apoptosis and chemosensitization in neuroblastomas cells. Eur Rev Med Pharmacol Sci 17: 2909-2915, 2013.

35. Tamm I, Wang Y, Sausville E, Scudiero DA, Vigna N, Oltersdorf T and Reed JC: IAP-family protein survivin inhibits caspase activity and apoptosis induced by Fas (CD95), Bax, caspases, and anticancer drugs. Cancer Res 58: 5315-5320, 1998.

36. Yamanaka K, Nakahara T, Yamauchi T, Kita A, Takeuchi M, Kiyonaga F, Kaneko N and Sasamata M: Antitumor activity of YM155, a selective small-molecule survivin suppressant, alone and in combination with docetaxel in human malignant melanoma models. Clin Cancer Res 17: 5423-5431, 2011.

37. Graf RP, Keller N, Barbero S and Stupack D: Caspase-8 as a regulator of tumor cell motility. Curr Mol Med 14: 246-254, 2014.

38. Feng W, Yoshida A and Ueda T: YM155 induces caspase- 8 dependent apoptosis through downregulation of survivin and Mcl-1 in human leukemia cells. Biochem Biophys Res Commun 435: 52-57, 2013. 